\title{
Are There MeV Gamma-Ray Bursts?
}

\author{
Tsvi Piran ${ }^{1,3}$ and Ramesh Narayan ${ }^{2,3}$ \\ 1. The Racah Institute for Physics, The Hebrew University, Jerusalem, Israel \\ 2. Harvard-Smithsonian Center for Astrophysics, Cambridge, MA, U.S.A. \\ 3. ITP, UCSB, Santa Barbara, , U.S.A.
}

\begin{abstract}
It is often stated that gamma-ray bursts (GRBs) have typical energies of several hundred $\mathrm{keV}$, where the typical energy may be characterized by the hardness $H$, the photon energy corresponding to the peak of $\nu F_{\nu}$. Among the 54 BATSE bursts analyzed by Band et. al. more than half have $100 \mathrm{keV}<H<400 \mathrm{keV}$. Is the narrow range of $H$ a real feature of GRBs or is it due to an observational bias? We consider the possibility that bursts of a given bolometric luminosity occur with a distribution: $p(H) d \log H \propto H^{\gamma} d \log H$. We model the detection efficiency of BATSE as a function of $H$ and calculate the expected distribution of $H$ in the observed sample for various values of $\gamma$. The Band sample shows a paucity of soft (X-ray) bursts, which may be real. However, because the detection efficiency of BATSE falls steeply with increasing $H$, the paucity of hard bursts need not be real. We find that the observed sample is consistent with a distribution above $H=100 \mathrm{keV}$ with $\gamma \approx 0$ (constant numbers of GRBs per decade of hardness) or even $\gamma=0.5$ (increasing numbers with increasing hardness). Thus, we suggest that a large population of unobserved hard gamma-ray bursts may exist. It is important to extend the present analysis to a larger sample of BATSE bursts and to include the OSSE and COMPTEL limits. If the full sample is consistent with $\gamma \gtrsim 0$, then it would be interesting to look for $\mathrm{MeV}$ bursts in the future.
\end{abstract}

One striking feature that is common to all gamma-ray bursts (GRBs) is the fact that most of the observed photons correspond to low energy gamma-rays, with energies of a few tens to few hundreds of $\mathrm{keV}$. While other features of the bursts, in particular the temporal structure, vary significantly from one burst to another, this feature seems to be quite invariant. One wonders, therefore, whether this is a clue to the nature of GRBs - a phenomenon that theorists should strive to explain - or if it is just the consequence of an observational bias against detection of harder or softer bursts.

Band et al. (11) have analyzed 54 strong GRBs (hereafter the Band sample), fitting the spectra using a four parameter function:

$$
N(E)= \begin{cases}\left(\frac{E}{100 \mathrm{keV}}\right)^{\alpha} \exp \left(-\frac{E}{E_{0}}\right) & \text { for }(\alpha-\beta) E<E_{0} ; \\ {\left[\frac{(\alpha-\beta) E_{0}}{100 \mathrm{keV}}\right]^{(\alpha-\beta)} \exp (\beta-\alpha)\left(\frac{E}{100 \mathrm{keV}}\right)^{\beta},} & \text { for } E>(\alpha-\beta) E_{0} .\end{cases}
$$

This function, which provides a good fit to most of the observed spectra,

(c) 1996 American Institute of Physics 
is characterized by two power laws joined smoothly at a break energy $H \equiv$ $(\alpha-\beta) E_{0}$. For most observed values of $\alpha$ and $\beta, \nu F_{\nu} \propto E^{2} N(E)$ rises below the break and decreases above it. The break energy $H$ is thus the "typical" energy of the observed radiation, in the sense that this is where the source emits the bulk of its luminosity. $H$ is correlated with, but not equal to, the hardness ratio which is commonly used in analyzing BATSE GRBs, namely the ratio of photons observed in channel 3 to those observed in channel 2.

Figure 1 shows the distribution of observed values of $H$ in the Band sample. There is a clear and marked maximum in the distribution for $H \sim 200 \mathrm{keV}$, and most of the bursts lie over the range $100 \mathrm{keV}<H<400 \mathrm{keV}$. Should we, therefore, conclude that most GRBs have hardnesses around 100-400 keV?

To answer this question we have used a simple model of the BATSE detector to calculate the expected hardness distribution of GRBs detected by BATSE for various assumed intrinsic hardness distributions. We have then compared the expected and observed distributions to see which intrinsic distributions are consistent with the data and which are not.

We assume that the intrinsic hardness distribution is as follows:

- All GRBs are adequately described by a Band spectrum, characterized by the parameters, $\alpha, \beta$, and $H$.

- The number of GRBs in a logarithmic interval, $d \log H$, varies as: $p(H) d \log H=p_{0} H^{\gamma} d \log H$.

- We also allow for the possibility that the intrinsic luminosity, $L$, is correlated with $H: L=L_{0}\left(H / H_{0}\right)^{\xi}$.

We now calculate, for given $\gamma$ and $\xi$, the distribution of $H$ values of bursts seen by a detector like BATSE. We make use of the fact that BATSE triggers on counts in the second and third energy channels, with photon energies between $50 \mathrm{keV}$ and $300 \mathrm{keV}$. We calculate, therefore, how many bursts with a given $H$ yield a count rate in the $50 \mathrm{keV}$ to $300 \mathrm{keV}$ band that is larger than a threshold, $C_{\min }$. (We ignore the fact that BATSE's counts in these channels may correspond to higher energy photons and that the intrinsic spectrum is related to the observed counts via the DRM, (2)). The count rate depends on $\alpha$ and $\beta$, in addition to the above mentioned dependence on $\xi$ and $\gamma$. We use the average values given by Band et al. (11): $\bar{\alpha}=-0.73$ and $\bar{\beta}=-2.22$. For reasons that will become clear shortly we also use $\tilde{\alpha}=-0.41$, which is the average value of $\alpha$ for GRBs with $50 \mathrm{keV}<H<300 \mathrm{keV}$.

The count rate in the $50-300 \mathrm{keV}$ energy band from a burst at a distance $D$ is:

$$
C=\frac{L(H) A}{4 \pi D^{2}} \mathcal{C}(50,300, \alpha, \beta, H)=\left[\frac{\left(L_{0} / H_{0}^{\xi}\right) A}{4 \pi D^{2}}\right] \mathcal{C}(50,300, \alpha, \beta, H) H^{\xi},
$$

where $A$ is the area of the detector. The quantity $\mathcal{C}$ represents the number of counts for every erg of energy incident on the detector (counts/ergs), and is 
easily calculated once the shape of the spectrum $(\alpha, \beta, H)$ and the detector limits $(50,300 \mathrm{keV})$ are given. The second equality follows from the assumed correlation between $L$ and $H$. For simplicity we use here and in the following a Newtonian geometry and ignore cosmological effects. This is justified since the Band sample is composed mostly of strong bursts for which cosmological redshift and spatial curvature effects are small. A detailed discussion that includes these effects will be published elsewhere (Cohen, Narayan \& Piran, 1996).

The number of bursts with hardness $H$ detected by a detector with a limiting sensitivity $C_{\min }$ is:

$$
N(H)=\left[\frac{4 \pi}{3}\left(\frac{\left(L_{0} / H_{0}^{\xi}\right) A}{4 \pi C_{\min }}\right)^{3 / 2} p_{0}\right] \mathcal{C}^{3 / 2}(50,300, \alpha, \beta, H) H^{3 \xi / 2+\gamma} .
$$

Figure 1 depicts $N(H)$, the expected number of detected bursts in a logarithmic interval of $H$, as a function of $\log (H)$. We have shown the calculated curves for $\alpha=\bar{\alpha}$ and $\alpha=\tilde{\alpha}$ and for $\gamma+3 \xi / 2=0$ and $\gamma+3 \xi / 2=0.5$; in all cases, we set $\beta=\bar{\beta}$. The observed distribution corresponding to the Band sample, with statistical error bars, is also shown on the same plot.

A comparison of the four calculated (or expected) curves with the observed distribution reveals immediately a paucity of soft bursts. In all four cases, the number of soft bursts we would have expected to see is significantly larger than the number actually observed. Therefore, unless BATSE has an unexpectedly large selection bias against detecting soft photons (i.e. significantly poorer sensitivity than we have assumed in our model), we conclude that the lower cut-off in the observed distribution of hardnesses is a real phenomenon. That is, there really are very few soft GRBs, and $N(H)$ does have a lower cutoff.

The story is, however, very different for larger values of $H$. The data show very small numbers of hard bursts; for instance, only two bursts out of 54 have $H>1 \mathrm{MeV}$. Nevertheless, this does not mean that GRBs intrinsically cut-off for hardnesses above $1 \mathrm{MeV}$. For instance, our theoretical model with $\xi=0$ and $\gamma=0$, with equal number of bursts per logarithmic hardness interval, actually predicts that even fewer bursts should have been observed. The best fit to the data is obtained with $\gamma+3 \xi / 2=0.5$ which corresponds to a burst population with an increasing number of bursts with hardness (for $\xi=0$ ) or to an equal number of bursts per logarithmic hardness interval $(\gamma=0)$ but with harder bursts being more luminous $(\xi=1 / 3)$.

The interpretation of the result is quite simple. There is an observational bias against detecting bursts with $H \gtrsim 500 \mathrm{keV}$ by current detectors. Two factors operate. For bursts with a fixed luminosity, harder bursts have fewer photons. This makes the detection of harder bursts more difficult in any detector that is triggered by photon counts. The decrease in sensitivity in BATSE is even more severe since BATSE triggers on photons in the $50 \mathrm{keV}$ to $300 \mathrm{keV}$ range and as the bursts becomes harder most of the emitted photons are further and further away from this energy range. 


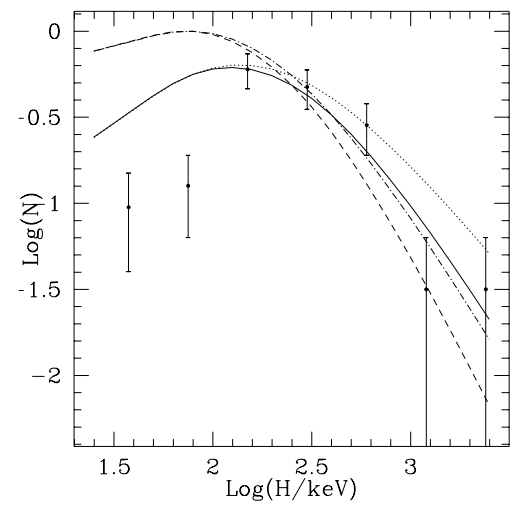

FIG. 1. Observed and expected numbers of detected bursts per interval of $\log (H)$. The the error bars represent statistical errors. The best fit (solid line) corresponds to $\alpha=\tilde{\alpha}=-0.41, \gamma+3 \xi / 2=0.5$. Other curves are: $\alpha=\bar{\alpha}=-0.7, \gamma+3 \xi / 2=0.5$ (dotted line), $\alpha=\tilde{\alpha}=-0.41, \gamma+3 \xi / 2=0$ (dashed line), and $\alpha=\bar{\alpha}=-0.7$, $\gamma+3 \xi / 2=0$ (dashed-dotted line).

The last point depends, of course, on the power law index $\alpha$ in the low energy range. It suggests that hard bursts that are detected by BATSE will have values of $\alpha$ lower than average. Fig. 2 depicts the distribution of $\alpha$ values in different hardness regimes, and shows the effect clearly. Among the 54 bursts in the Band sample, hard bursts do have significantly more negative values of $\alpha$. It is for this reason that we have introduced $\tilde{\alpha}$, the average $\alpha$ value over the intermediate hardness range, $50 \mathrm{keV}<H<300 \mathrm{keV}$. If the intrinsic $\alpha$ distribution is independent of the hardness than $\tilde{\alpha}$ is a better estimate of the average $\alpha$ than $\bar{\alpha}$.

Figure 2, by itself, without any of the theoretical arguments presented earlier, suggests the existence of the selection effect we have discussed. There are two ways of interpreting the evidence in this plot. One could say that the plot reflects the true distribution of burst properties and that for some reason $\alpha$ happens to be correlated with $H$ in the particular manner seen in the data. This is very ad hoc. The alternative, which we find much more attractive, is to say that BATSE has difficulty detecting hard bursts, and finds it particularly difficult to detect hard bursts with less negative, or positive, values of $\alpha$; that is, such bursts do exist, but BATSE misses them. If we accept the latter explanation, then it means that BATSE is certainly missing at least some part of the hard population, namely those bursts with less negative (or positive) $\alpha$. It is then but a small step to accept the entire argument presented earlier.

Our main result then is the following. When observational selection effects are taken into account, the two hard bursts with $H>1 \mathrm{MeV}$ in the Band sample are but "the tip of the iceberg," and represent a large number of 


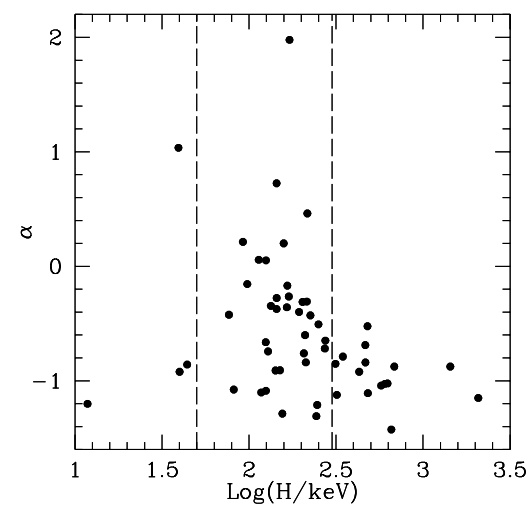

FIG. 2. The low energy spectral slope $\alpha$ vs. the hardness $H$ in the Band sample. Hard bursts tend to have more negative $\alpha$, which we interpret as evidence for the selection effect discussed in the paper. The vertical lines mark $50 \mathrm{keV}$ and $300 \mathrm{keV}$

undetected hard GRBs. The observed distribution of hardness is, in fact, consistent with the possibility that most GRBs are harder than the bursts detected by BATSE, and that there is a large population of mostly undetected GRBs whose typical photon energy is in the $\mathrm{MeV}$, or even harder, range.

So far we have compared the theoretical predictions to the hardness distribution in the Band sample. It will be interesting to perform the same analysis on a larger sub-sample (see (3)). It will also be of great interest to perform a similar analysis on data from other GRB detectors. One intriguing possibility is to search in BATSE's raw channel 4 data for untriggered events, which might be some of the missing hard bursts. This would be a complementary search to the soft GRB search reported by Kommers et. al. (4). In the meantime, before we know whether hard bursts exist or not, we should be very cautious about performing correlations between various characteristics of the observed bursts and their hardness parameters. The data are biased, as far as $\mathrm{H}$ is concerned, and any correlation seen (e.g. the correlation between $\alpha$ and $H$ in Fig. 2) might reflect nothing more than selection effects in the data.

We thank B. Paczński for remarks that motivated this research. The research was supported by a NASA grant NAG5-1904 to Harvard University by a Basic research grant to the Hebrew University and by NSF grant PHY9407194 to the ITP.

\section{REFERENCES}

1. Band, D., et. al., 1993, Ap. J. 413, 281.

2. Pendleton et. al. 1995, preprint.

3. Cohen, E., Narayan, R., \& Piran, T., 1996, in preparation. 
4. Kommers, J.M., Lewin, W.H.G., van Paradijs, J. Kouveliotou C. \& Fishman G.J, 1996, this conference. 